\title{
In vivo characterization of developing chronic pancreatitis in rats
}

\author{
Claudia Glawe ${ }^{1}$, Jörg Emmrich ${ }^{2}$, Gisela Sparmann² and Brigitte Vollmar ${ }^{1}$ \\ ${ }^{1}$ Department of Experimental Surgery, University of Rostock, Rostock, Germany and ${ }^{2}$ Department of \\ Gastroenterology, University of Rostock, Rostock, Germany
}

\begin{abstract}
Despite numerous experimental and clinical investigations, there is no unifying concept on pathophysiology and pathogenesis of chronic pancreatitis. Defining the interplay between pancreatic microcirculation and parenchymal tissue, we will provide a basis for the better understanding of pancreatic fibrogenesis using in vivo high-resolution multifluorescence microscopy in dibutyltin chloride (DBTC)-exposed rats. Pancreatic microcirculation at days 3 and 7 after DBTC revealed leukocyte activation with a two-fold higher fraction of rolling cells and a nine- to 10-fold increase of cells firmly adherent to the endothelial lining, followed by subsequent transendothelial migration into tissue, as given by chloracetate esterase histology. In vivo staining of acinar tissue with bisbenzimide presented single cells exhibiting nuclear chromatin condensation and fragmentation. Apoptotic cell death was confirmed by immunohistochemical staining for active caspase-3 as well as by TUNEL analysis. Necrotic cells were found dispersed throughout the exocrine tissue under observation. Both modes of cell death were found highest in extent at days 3 and 7 with $15-20 \mathrm{cells} / \mathrm{mm}^{2}, \mathrm{but}$ progressively decreased below $10 \mathrm{cells} / \mathrm{mm}^{2}$ up to 28 days after DBTC. By means of in vivo microscopy yellowgreen autofluorescent collagen deposits were found at day 7 and progressively increased up to $\sim 12 \%$ at day 28 after DBTC. Concomitantly, density of capillaries progressively decreased and capillaries failing to conduct blood flow became apparent. Present on-line analysis indicates an early inflammatory response with acinar cell death, most probably triggering progression of disease with collagen deposition, capillary rarefication and manifestation of perfusion failure. These temporal and spatial multiparameter measurements of the in vivo microenvironment provide new insights into the pathological processes of pancreatic fibrogenesis.

Laboratory Investigation (2005) 85, 193-204, advance online publication, 15 November 2004; doi:10.1038/labinvest.3700212
\end{abstract}

Keywords: apoptosis; autofluorescence; caspase-3; collagen; inflammation; necrosis; nutritive perfusion; organ remodeling

Chronic pancreatitis is a progressive disease characterized by irreversible destruction of exocrine pancreatic tissue and extensive fibrosis. Despite the identification and characterization of stellate cells as potential effector cells in pancreatic fibrosis, ${ }^{1-4}$ the understanding of the pathogenesis of chronic pancreatitis is still limited, partly due to the lack of appropriate animal models. Several theories, most notably the 'toxic-metabolic' or the 'protein plug', have been suggested in the past, ${ }^{5,6}$ but could not be unequivocally confirmed by experimental data. Another concept based on relapsing acute hemorrhagic-necrotizing pancreatitis was presented by Klöppel and Maillet. ${ }^{6-8}$ Strong support for this 'necrosis-fibrosis' concept came from morphological data in a clinical long-term study, implying

Correspondence: Dr B Vollmar, MD, Department of Experimental Surgery, University of Rostock, Schillingallee 70, D-18055 Rostock, Germany

E-mail: brigitte.vollmar@med.uni-rostock.de

Received 28 August 2004; revised and accepted 11 October 2004; published online 15 November 2004 an evolution from severe acute pancreatitis to chronic pancreatitis. ${ }^{9}$

While microcirculatory deteriorations are well established as important in both the initiation and progression of acute pancreatitis, ${ }^{10-12}$ the pancreatic microcirculation has not systematically been studied during development of chronic pancreatitis. Thus, the implication of observations, such as decreased pancreatic blood flow during chronic disease,$^{13}$ is unclear. Microangiography has proved useful in demonstrating changes of the vasculature in pancreatic fibrosis; ${ }^{14,15}$ however, this technique is suited neither for the investigation of dynamic alterations in the microvascular function nor for the direct assessment of the close inter-relation between the pancreatic microcirculation, parenchymal cell integrity and organ function.

In addition to acute pancreatitis, the organotin dibutyltin dichloride (DBTC) has been reported to induce a chronic course of inflammation accompanied by the development of pancreatic fibrosis in rats in dependence on the time and dose after single treatment of the animals. ${ }^{16,17}$ Thus, this model seems 
to be ideally suited to combine several applications of intravital multifluorescence microscopy for the comprehensive assessment of microvascular, cellular and morphological aspects during the process of pancreatic fibrogenesis.

\section{Materials and methods}

\section{Animal Model}

After approval by the local animal welfare committee (LVL M-V/310-4/7221.3-1.1-046/03), experiments were performed in accordance with the German legislation on protection of animals and the 'Guide for the Care and Use of Laboratory Animals (NIH publication no. 86-23, revised 1985). Under brief ether anesthesia, 28 male Lewis rats (Charles River, Fa. Wiga, Sulzfeld, Germany) weighing 160-180g were injected with dibutyltin dichloride (DBTC, $8 \mathrm{mg} / \mathrm{kg}$, dissolved in 100\% ethanol/glycerol $2: 5 \mathrm{v} /$ v; Sigma-Aldrich, Deisenhofen, Germany) via the tail vein, as previously described. ${ }^{17}$ Animals that received equivalent volumes of solvent only served as vehicle-treated controls (vehicle, $n=12$ ). Additional animals without any intervention served as untreated controls (control, $n=3$ ). After awakening, animals were kept one per cage on a standard light cycle and had free access to standard laboratory chow and water ad libitum.

\section{In Vivo Studies}

At 3, 7, 14 and 28 days after the application of DBTC ( $n=7$ animals per time point) or solvent ( $n=3$ per time point), intravital fluorescence microscopy of the pancreas was performed. For this purpose, animals were anesthetized with pentobarbital ( $50 \mathrm{mg} / \mathrm{kg}$ body wt ip) and placed in supine position on a heating pad. After tracheotomy to facilitate spontaneous breathing, the right carotid artery was exposed and cannulated for heart rate and blood pressure monitoring (PE-50; ID $0.58 \mathrm{~mm}$; Portex, Hythe, UK). An additional catheter (PE-50) in the right jugular vein served as a route for administration of fluorescent tracers. After transverse laparotomy and dissection of the omentum from the greater curvature of the stomach, the tail and parts of the corpus of the pancreas were gently exteriorized on a plasticine stage, allowing ideal placement of the tissue, which guaranteed adequate homogeneous focus level and minimal respiratory movement for the microscopic procedure. ${ }^{12}$ To keep the exteriorized pancreas moist and to exclude effects of ambient oxygen, the pancreatic surface was covered with an oxygen-impermeable Saran wrap. ${ }^{12}$

\section{Intravital Fluorescence Microscopy}

Using a Zeiss Axiotech microscope equipped with a 100 W HBO mercury lamp and different filter blocks for epi-illumination (Zeiss, Oberkochen, Germany; blue: $450-490 />520 \mathrm{~nm}$ excitation/emission wavelength, green: $530-560 />580 \mathrm{~nm}$, and ultraviolet: $330-380 />415 \mathrm{~nm}$ ), microscopic images were recorded by a charge-coupled device video camera (FK 6990-IQ, Pieper, Berlin, Germany) and transferred to a video system for off-line evaluation. With the use of $\times 10, \times 20$, and $\times 40$ objectives (Zeiss), magnifications of $\times 208, \times 418$, and $\times 807$ were achieved on a 17 -in video screen.

Using blue fluorescence epi-illumination, microfluorographic images of autofluorescence of the pancreatic surface (10 observation areas (1226× $917 \mu \mathrm{m}^{2}$ ) per animal) were taken, which allowed for detection of extracellular matrix composites due to emission maxima of collagen above $435 \mathrm{~nm} \cdot{ }^{18-20}$ Subsequently, contrast enhancement for assessment of capillary perfusion was achieved by intravenous injection of $2 \%$ fluorescein-isothiocyanate (FITC)labeled dextran (MW 150000; $0.1 \mathrm{ml} / 100 \mathrm{~g}$ body weight; Sigma, Deisenhofen, Germany) and blue light epi-illumination. Leukocyte-endothelial cell interaction was documented after in vivo staining of white blood cells by $1 \%$ rhodamine- $6 \mathrm{G}(0.1 \mathrm{ml} / 100 \mathrm{~g}$ body weight iv) and green light epi-illumination. Intravital microscopic analyses of the microcirculation included the determination of capillary diameter, red blood cell (RBC) velocity in capillaries (RBC-velocity ${ }_{\text {сар }}$ ), functional capillary density (FCD) as a parameter of nutritive perfusion and heterogeneity of nutritive perfusion. ${ }^{12,21}$ Moreover, we assessed microvascular leukocyte count and flow behavior of leukocytes in postcapillary venules, classified according to their interaction with the endothelial lining as adherent, rolling or freeflowing (nonadherent) cells. ${ }^{12,22}$ For analysis of apoptotic cell death, in vivo staining of acinar cell nuclei was achieved by intravenous injection of $1 \%$ bisbenzimide (Hoechst 33342; $0.1 \mathrm{ml} / 100 \mathrm{~g}$ body weight; Sigma) and ultraviolet epi-illumination. ${ }^{23-25}$ Intravenously applied $1 \%$ propidium iodide $(0.1 \mathrm{ml} / 100 \mathrm{~g}$ body weight $)$ served for staining of necrotic acinar cells by failure of exclusion of the dye due to irreversible cell damage. ${ }^{26}$

\section{Quantitative Microcirculatory Analysis}

For assessment of collagen, autofluorescent structures were determined planimetrically with subsequent automatic calculation of the area of positive deposits in percent of the whole area of observation $\left(1226 \times 917 \mu \mathrm{m}^{2}\right)$. Capillary diameter $(\mu \mathrm{m})$ was assessed in 10 individual capillaries per observation field and 10 observation fields per animal. Within these capillaries, RBC-velocity cap $_{\text {was }}$ analyzed using the line-shift method. ${ }^{27}$ FCD (given as $\mathrm{cm} / \mathrm{cm}^{2}$ ) was defined as the length of all RBCperfused nutritive capillaries per observation area $\left(614 \times 462 \mu \mathrm{m}^{2}\right)$ and assessed in 10 randomly selected observation areas using a computer-assisted 
image analysis system (CapImage; Zeintl, Heidelberg, Germany). To assess the degree of nutritive perfusion inhomogeneities, the coefficient of variation (relative dispersion) of FCD was calculated by the formula: $\mathrm{CV}_{\mathrm{FCD}}=$ s.d. $/ \mathrm{FCD}_{\text {mean }} \times 100(\%)$, where s.d. represents the standard deviation and $\mathrm{FCD}_{\text {mean }}$ the mean value of FCD obtained from all observation areas. $^{12}$ To address aspects of organ remodeling, density of capillaries was assessed by counting the number of microvessels irrespective of conductance of flow, which intersected a line $200 \mu \mathrm{m}$ in length. ${ }^{20}$

In postcapillary venules, leukocyte flux was determined by counting all cells passing the vessel segment under investigation. Adherent leukocytes were defined in each vessel segment as cells that did not move or detach from the endothelial lining within an observation period of $20 \mathrm{~s}$ and are given as number of cells per $\mathrm{mm}^{2}$ of endothelial surface, calculated from diameter and length $(100 \mu \mathrm{m})$ of the vessel segment studied, assuming cylindrical geometry. Rolling leukocytes were defined as those white cells moving at a velocity less than $2 / 5$ of that of erythrocytes in the centerline of the microvessel and are given as percentage of nonadherent leukocytes passing through the observed vessel segment within $30 \mathrm{~s}^{12,22}$ Apoptotic cell death was analyzed within 10 observation fields per animal by counting the number of cells which showed apoptosisassociated condensation, fragmentation and crescent-shaped formation of chromatin (given as cells/ $\mathrm{mm}^{2}$ observation field). ${ }^{23-25}$ Necrosis was assessed by counting the number of propidium iodide positive cells within 10 observation fields (given as cells $/ \mathrm{mm}^{2}$ observation field). ${ }^{26}$

\section{Routine Histology and Immunohistochemistry}

At the end of each experiment, pancreatic tissue was fixed in $4 \%$ phosphate-buffered formalin for $2-3$ days and then embedded in paraffin. From the paraffin-embedded tissue blocks, $4 \mu \mathrm{m}$ sections were cut and stained for conventional tinctorial stains, that is, hematoxylin-eosin for routine histology and a trichromatic stain for assessment of interstitial tissue (Ladewig staining). Interstitial tissue was scored in 50 high-power fields (HPF; $\times 400$ magnification) from 0 to 4 , where none and minimal $(<10 \%$ of the observation area $)=1$; mild $(10-30 \%$ of the observation area) $=2$; moderate $(30-50 \%$ of the observation area) $=3$ and severe $(>50 \%$ of the observation area) $=4$. Leukocytes were stained by the AS-D chloroacetate esterase (CAE) technique and were identified by positive staining and morphology in 50 consecutive HPF.

To confirm apoptotic cell death, we studied active caspase- 3 by immunohistochemistry. For this purpose, $4 \mu \mathrm{m}$ sections of paraffin-embedded pancreatic tissue were mounted on poly-L-lysine slides and incubated overnight at room temperature with a rabbit polyclonal anticleaved-caspase-3 antibody
(1:25; Cell Signaling Technology, Frankfurt, Germany). This antibody detects endogenous levels of the large fragment $(17 / 19 \mathrm{kDa})$ of activated caspase-3, but not full-length caspase-3. A horseradish peroxidase-conjugated anti-rabbit/anti-mouseIg-antibody was used as secondary antibody (Dako Envision Link, Doublestain system, Hamburg, Germany). 3,3' diaminobenzidine was used as chromogen. The sections were counterstained with hemalaun and caspase-3-positive cells were counted within 50 HPF (given as cells per HPF). In addition, $4 \mu \mathrm{m}$ sections of pancreatic tissue were exposed to an apoptosis-specific staining kit (indirect in situ DNA nick end labeling (TUNEL) assay; ApopTag, CHEMICON International, Inc., Temecula, CA, USA) according to the manufacturer's instructions. Quantitative analysis was performed by counting the number of TUNEL positive tissue-confined cells within $50 \mathrm{HPF}$ (given as cells per HPF).

The spatial relationship of activated pancreatic stellate cells to pancreatic fibrosis was studied by dual staining for alpha-smooth muscle actin (SMA) plus Sirius Red. Paraffin sections of the pancreas were prepared as described before and then incubated at room temperature for $30 \mathrm{~min}$ with the mouse monoclonal anti-SMA primary antibody (1:400; Sigma, Taufkirchen, Germany). A horseradish peroxidase-conjugated anti-rabbit/anti-mouseIg-antibody was used as secondary antibody (Dako Envision Link, Doublestain system, Hamburg, Germany). 3,3' diaminobenzidine was used as chromogen. The sections were further processed by incubation with Sirius Red stain (Direct Red 80, Sigma) for $1 \mathrm{~h}$ at room temperature, followed by counterstaining with hemalaun.

\section{Serum Parameters}

After intravital microscopic analysis of the pancreatic microcirculation, arterial blood samples were taken for spectrophotometric determination of serum lipase, amylase and lactate dehydrogenase $(\mathrm{LDH})$ activities.

\section{Statistics}

All values are expressed as means \pm s.e.m. Since no significant difference was measured in any of the evaluated parameters between the vehicle-treated animals at different time points, these were grouped together for graphic representation and statistical evaluation. Values of untreated control animals, which served as physiological values and are displayed in the graphs, however, were not considered for statistical analysis between groups. After proving the assumption of normality and homogeneity of variance across groups, differences between the DBTC-exposed groups and the vehicletreated control group were calculated using the unpaired Student's $t$-test, including correction of the 
$\alpha$-error according to the Bonferroni probabilities for repeated analysis (SigmaStat, Jandel Corporation, San Rafael, CA, USA). The criterion for significance was taken to be $P<0.05$.

\section{Results}

\section{Gross Findings}

During the first week upon DBTC exposure, animals did not gain weight $(-1.3 \pm 3.1 \%)$ when compared to vehicle-treated control animals $(+7.9 \pm 0.2 \%)$. Moreover, final gain weight in the DBTC-exposed animals over the 28-day observation period was found to be significantly lower $(+23.3 \pm 1.9 \%$, $P<0.05$ vs vehicle: $+63.0 \pm 6.9 \%$ ). At gross inspection after laparotomy, pancreatic tissue at 14 and 28 days after DBTC presented with dilatation of the pancreatic duct and marked atrophy in contrast to normal macroscopic appearance of pancreatic glands in vehicle-treated control animals. While DBTC-exposed animals did not differ from vehicletreated animals with respect to hemodynamics, they did show systemic leuko- and thrombocytopenia at day 3 (Table 1). Depletion of systemic blood cell counts was followed by supranormal values at days 7 and 14 after DBTC exposure (Table 1).

\section{Intravital Microscopic Analysis}

Coincident with systemic leukocytopenia, pancreatic microcirculation at early time points after DBTC was characterized by intrapancreatic leukocyte accumulation with considerably high numbers of cells interacting with the endothelial lining. As assessed on days 3 and 7, DBTC caused a two-fold increase in the fraction of rolling leukocytes (Figure 1a) and a nine- to 10 -fold increase in the number of firmly adherent leukocytes (Figure 1b). Calculation

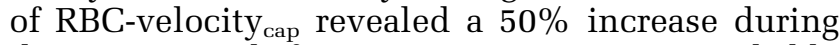
this time period after DBTC exposure, most probably reflecting an inflammation-associated hyperperfusion (Table 2).

Interaction of leukocytes with the microvascular endothelium was followed by transendothelial migration and massive tissue infiltration, as given by the eight-fold increase of CAE-stained leukocytes in pancreatic tissue at day 7 after DBTC (Figure 2).

Although still significantly higher than in vehicletreated control animals, venular leukocyte adherence and tissue infiltration were found markedly lower at days 14 and 28 when compared with early stages after DBTC exposure (Figure 1b). However, maintenance of increased rolling fraction of leukocytes at 14-28 days after DBTC implied that inflammatory stimulus is strong enough to sustain loose interaction of cells with the endothelium (Figure 1a).

Analysis of acinar cell morphology/integrity by in vivo fluorescence microscopy showed a significant rise of apoptotic cells from less than 5 cells $/ \mathrm{mm}^{2}$ under control conditions to $21 \pm 2$ and $17 \pm 2$ cells/ $\mathrm{mm}^{2}$ at days 3 and 7 after DBTC with a $50 \%$ decrease to approximately 9 cells $/ \mathrm{mm}^{2}$ at later time points after DBTC exposure (Figure 3). Analysis of pancreatic necrosis revealed a comparable pattern with the highest values at these early time points (Figure 4). Of interest, the majority of cells showing apoptosis-associated condensation, fragmentation and/or crescent-shaped formation of nuclear chromatin exhibited propidium iodide staining, indicative of concomitant necrosis-associated cell membrane damage (Figure 5).

Intravital observation of the pancreatic autofluorescence (330-390/> $430 \mathrm{~nm}$ ) revealed an inhomogeneous distribution of nonfading autofluorescence throughout the tissue under investigation, consistent with collageneous deposits (Figure 6). Quantitative analysis of the area of collagen autofluorescence in chronic pancreatitis revealed a progressive $(P<0.05$ vs control) increase to $1.4 \pm 1.4$, $9.9 \pm 1.4$ and $12.4 \pm 0.9 \%$ from days 7 to 28 after DBTC exposure, whereas collagen autofluorescence

Table 1 Systemic hemodynamics, blood cell count and hematocrit in animals at 3, 7, 14 and 28 days after intravenous DBTC exposure

\begin{tabular}{|c|c|c|c|c|c|c|}
\hline & \multirow[t]{2}{*}{ Control } & \multirow[t]{2}{*}{ Vehicle } & \multicolumn{4}{|c|}{ After DBTC exposure } \\
\hline & & & Day 3 & Day 7 & Day 14 & Day 28 \\
\hline MAP (mmHg) & $110 \pm 3$ & $101 \pm 6$ & $86 \pm 8$ & $82 \pm 5$ & $91 \pm 6$ & $85 \pm 9$ \\
\hline Heart rate $\left(\min ^{-1}\right)$ & $328 \pm 33$ & $329 \pm 12$ & $304 \pm 13$ & $300 \pm 21$ & $300 \pm 17$ & $289 \pm 24$ \\
\hline Leukocytes $\left(\times 10^{9} / \mathrm{l}\right)$ & $6.4 \pm 0.4$ & $5.5 \pm 0.5$ & $2.9 \pm 0.5^{*}$ & $8.1 \pm 0.6^{*}$ & $8.9 \pm 1.0^{*}$ & $6.7 \pm 1.8$ \\
\hline Thrombocytes $\left(\times 10^{9} / 1\right)$ & $786 \pm 90$ & $837 \pm 30$ & $511 \pm 61^{*}$ & $1254 \pm 100^{*}$ & $1168 \pm 97^{*}$ & $834 \pm 87$ \\
\hline Hematocrit (\%) & $42 \pm 1$ & $50 \pm 1$ & $41 \pm 2^{*}$ & $49 \pm 1$ & $44 \pm 7$ & $47 \pm 2$ \\
\hline
\end{tabular}

Animals that received equivalent volumes of solvent (vehicle) and animals without any intervention (control) served as controls.

Values are given as mean \pm s.e.m. DBTC animals, $n=7$ per time point; vehicle-treated animals, $n=12$, untreated control animals, $n=3$. ${ }^{\star} P<0.05$ vs vehicle. 

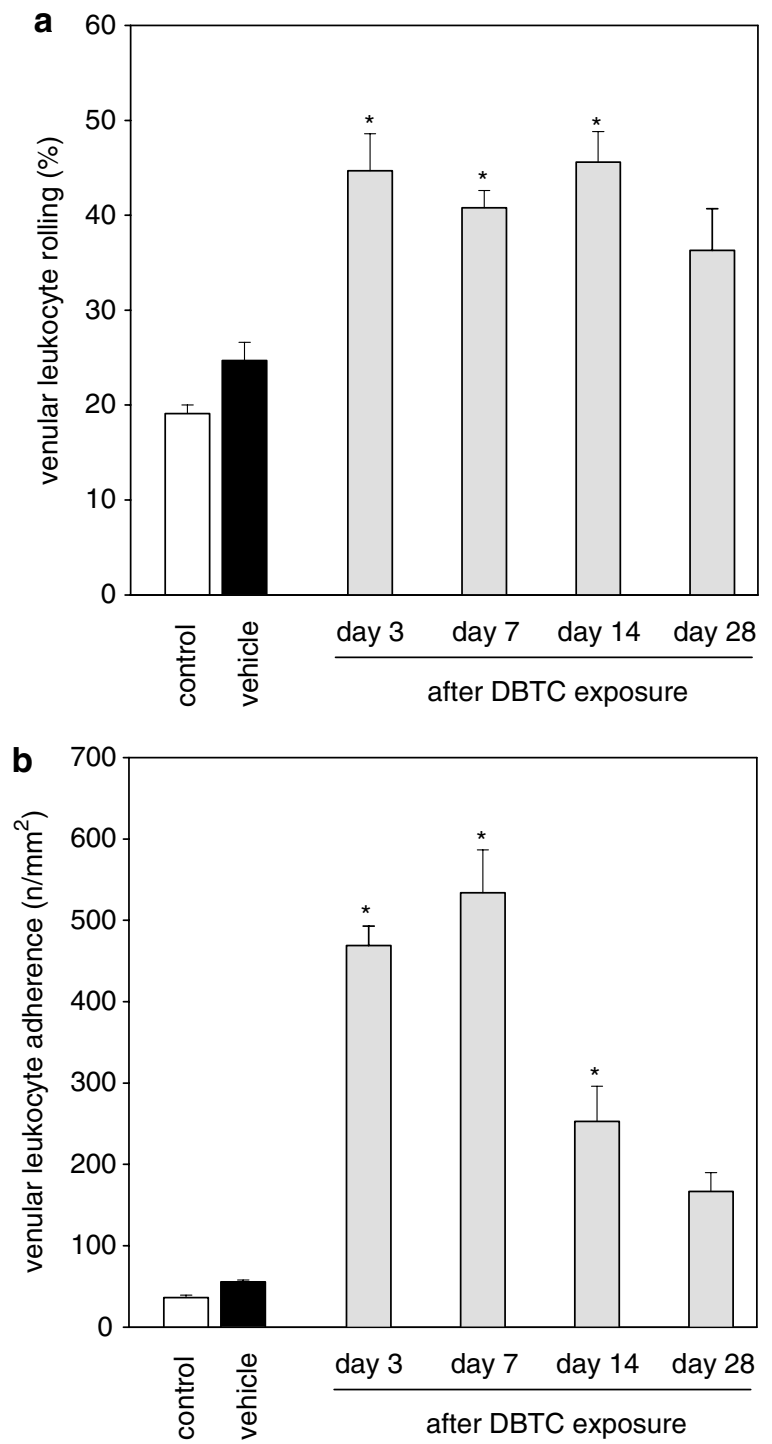

Figure 1 Quantitative analysis of venular leukocyte-endothelial cell interaction, that is, the fraction of rolling leukocytes (a) and the number of firmly adherent leukocytes (b) within postcapillary venules of pancreatic tissue in animals at 3, 7, 14 and 28 days after intravenous DBTC exposure ( $n=7$ per time point). Animals that received equivalent volumes of solvent (vehicle, $n=12$ ) and animals without any intervention (control, $n=3$ ) served as controls. Using intravital fluorescence microscopy and a computer-assisted analysis system for off-line analysis, measurements were performed in green epi-illumination after injection of rhodamine $6 \mathrm{G}$ for in vivo staining of leukocytes. Mean \pm s.e.m., ${ }^{*} P<0.05$ vs vehicle.

was not detectable in pancreatic glands of either control animals or animals at day 3 after DBTC exposure (Figure 6). Collagen deposition was paralleled by a decrease of capillary density (Figure 7a). Besides capillary rarefication, perfusion failure occurred, as given by the decrease in FCD from approximately $400 \mathrm{~cm} / \mathrm{cm}^{2}$ to $310 \pm 8$ and $301 \pm$ $9 \mathrm{~cm} / \mathrm{cm}^{2}$ at 14 and 28 days after DBTC exposure (Figure 7b). Moreover, calculation of the $\mathrm{CV}_{\mathrm{FCD}}$ indicated a high degree of perfusion inhomogene-
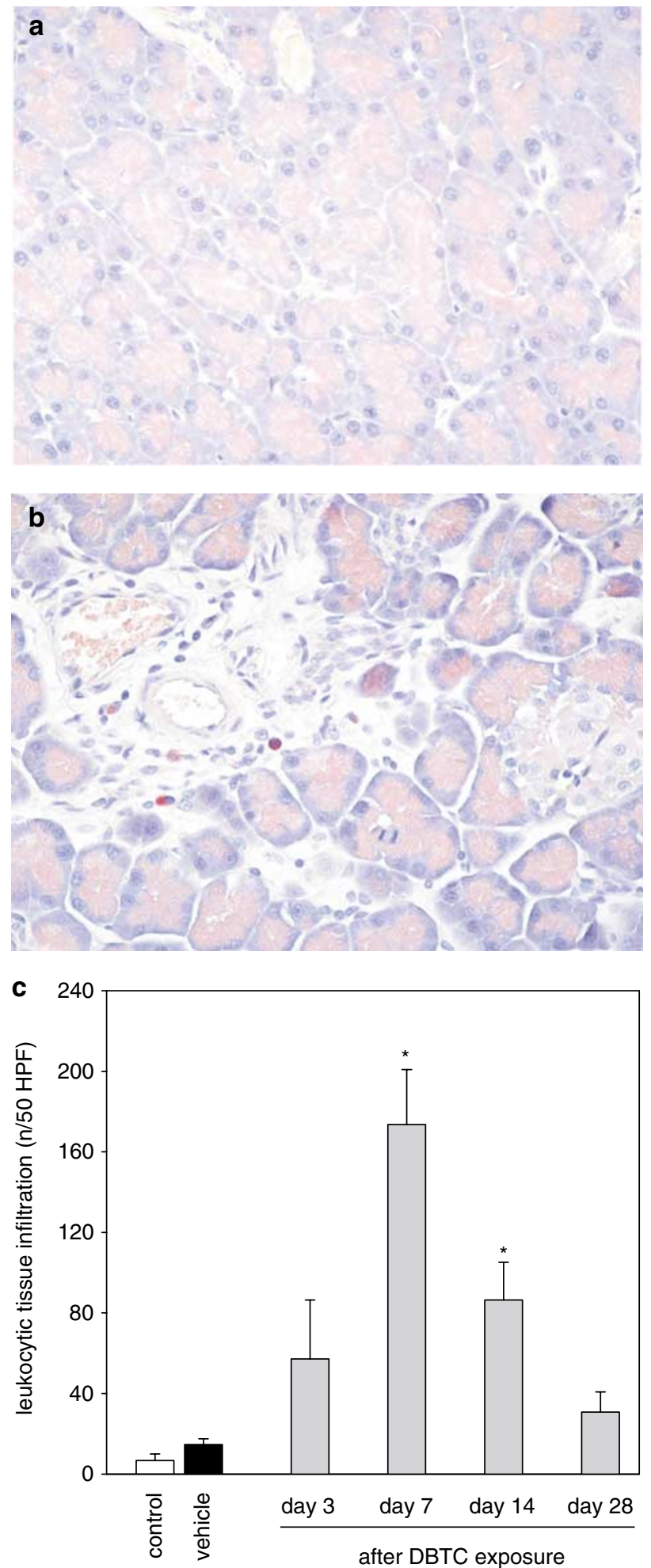

Figure 2 Representative images of leukocytic infiltration of pancreatic tissue after (a) vehicle-treatment or (b) at day 7 after DBTC exposure. Original magnification $\times 400$. (c) Quantitative analysis of CAE-positive leukocytes (given as cells per $50 \mathrm{HPF}$ ) infiltrating the pancreatic tissue in animals at 3, 7, 14 and 28 days after intravenous DBTC exposure ( $n=7$ per time point). Animals that received equivalent volumes of solvent (vehicle, $n=12$ ) and animals without any intervention (control, $n=3$ ) served as controls. Mean \pm s.e.m., ${ }^{*} P<0.05$ vs vehicle. 
Table 2 Capillary red blood cell velocity (RBC-velocity ${ }_{\text {сap }}$ ), coefficient of variation of functional capillary density (CV $\mathrm{FCD}_{\mathrm{FCD}}$ and capillary diameter in animals at 3, 7, 14 and 28 days after intravenous DBTC exposure

\begin{tabular}{|c|c|c|c|c|c|c|}
\hline & \multirow[t]{2}{*}{ Control } & \multirow[t]{2}{*}{ Vehicle } & \multicolumn{4}{|c|}{ After DBTC exposure } \\
\hline & & & Day 3 & Day 7 & Day 14 & Day 28 \\
\hline RBC-velocity ${ }_{\text {cap }}(\mu \mathrm{m} / \mathrm{s})$ & $413 \pm 9$ & $431 \pm 17$ & $480 \pm 58$ & $654 \pm 58^{*}$ & $403 \pm 26$ & $408 \pm 28$ \\
\hline $\begin{array}{l}\mathrm{CV}_{\mathrm{FCD}} \\
\text { Capillary diameter (um) }\end{array}$ & $3.3 \pm 1.1$ & $2.3 \pm 0.5$ & $4.3 \pm 1.1$ & $3.8 \pm 1.2$ & $7.6 \pm 0.8^{*}$ & $7.4 \pm 1.2^{*}$ \\
\hline Capillary diameter $(\mu \mathrm{m})$ & $7.3 \pm 0.2$ & $7.9 \pm 0.1$ & $8.5 \pm 0.6$ & $8.0 \pm 0.5$ & $8.8 \pm 0.1^{*}$ & $9.0 \pm 0.1^{*}$ \\
\hline
\end{tabular}

Animals that received equivalent volumes of solvent (vehicle) and animals without any intervention (control) served as controls.

Values are given as means \pm s.e.m. DBTC animals, $n=7$ per time point; vehicle-treated animals, $n=12$, untreated control animals, $n=3$. ${ }^{*} P<0.05$ vs vehicle.

Table 3 Serum activities of lipase, amylase and lactate dehydrogenase (LDH) in animals at 3, 7, 14 and 28 days after intravenous DBTC exposure

\begin{tabular}{|c|c|c|c|c|c|c|}
\hline & \multirow[t]{2}{*}{ Control } & \multirow[t]{2}{*}{ Vehicle } & \multicolumn{4}{|c|}{ After DBTC exposure } \\
\hline & & & Day 3 & Day 7 & Day 14 & Day 28 \\
\hline Lipase (U/l) & $14 \pm 3$ & $13 \pm 2$ & $243 \pm 91^{*}$ & $27 \pm 14$ & $18 \pm 2$ & $15 \pm 2$ \\
\hline Amylase (U/l) & $2650 \pm 461$ & $2175 \pm 74$ & $3753 \pm 1021$ & $1432 \pm 227$ & $1724 \pm 181$ & $2589 \pm 185$ \\
\hline LDH (U/l) & $311 \pm 108$ & $441 \pm 35$ & $1021 \pm 299$ & $1127 \pm 488$ & $886 \pm 269$ & $619 \pm 91$ \\
\hline
\end{tabular}

Animals that received equivalent volumes of solvent (vehicle) and animals without any intervention (control) served as controls.

Values are given as means \pm s.e.m. DBTC animals, $n=7$ per time point; vehicle-treated animals, $n=12$, untreated control animals, $n=3$. ${ }^{*} P<0.05$ vs vehicle.

ities (Table 2). Additionally, capillaries were found enlarged during the later course of DBTC pancreatitis (Table 2).

\section{Laboratory Analyses}

Analysis of serum lipase activities demonstrated significant increases at day 3 with return to almost physiological values at days 7,14 and 28, indicating distinct parenchymal cell damage during the early period upon DBTC exposure (Table 3). Like lipase, amylase activities showed a comparable pattern, however, without statistical significant differences due to high variation (Table 3 ). In addition, LDH values reflected overall organ injury during the course of pancreatic fibrogenesis (Table 3).

\section{Histology and Immunohistochemistry}

To confirm apoptotic cell death after DBTC exposure, active caspase-3 was studied by immunohistochemistry (Figure 8). These experiments showed positive staining of individual acinar cells with highest values at 7 and 14 days (Figure 8). Correspondingly, TUNEL staining of pancreatic tissue revealed an increase of positive cells from $0.1 \pm 0.1$ cells $/ \mathrm{HPF}$ in controls to $1.1 \pm 0.1,1.9 \pm 1.3$ and $2.0 \pm 0.3$ cells/HPF after 3,7 and 14 days, respectively, confirming the apoptotic mode of cell death during early stages in DBTC pancreatitis. At day 28, apoptosis was found reduced with only $0.5 \pm 0.2$ TUNEL positive cells/HPF.

In line with kinetics of collagen deposition, Ladewig staining confirmed the microscopically assessed increase of interstitial tissue with a rise of the scores from $<2$ in vehicle-treated controls to almost 3 over the 28-day course of DBTC. Additionally, dual staining technique of pancreatic tissue at 7 , but in particular at 14 and 28 days after DBTC exposure revealed a strong association between activated stellate cells and fibrosis, as given by the close vicinity of Sirius Red collagen staining and SMA-positive cells (Figure 9).

\section{Discussion}

Etiology of human chronic pancreatitis comprises alcoholic, hereditary, autoimmune, idiopathic, tropical and other rare types, each differing in phenoptype of fibrosis. ${ }^{28}$ Regardless of the fibrosis pattern, sequelae of basic processes have been postulated $;^{28}$ however, so far they have not conclusively been shown. This comprehensive analysis now provides for the first time in vivo evidence for a process of pancreatic fibrogenesis, as demonstrated for other organs. Multiparameter assessment by in vivo fluorescence microscopy of the pancreas indicates an early inflammatory response upon DBTC exposure with a concomitant increase in both apoptotic and necrotic death of acinar cells, 
followed by collagen deposition, microvessel rarefication and, thus, organ remodeling.

In vivo fluorescence microscopy of bisbenzimidestained parenchymal tissue and immunohistochem-
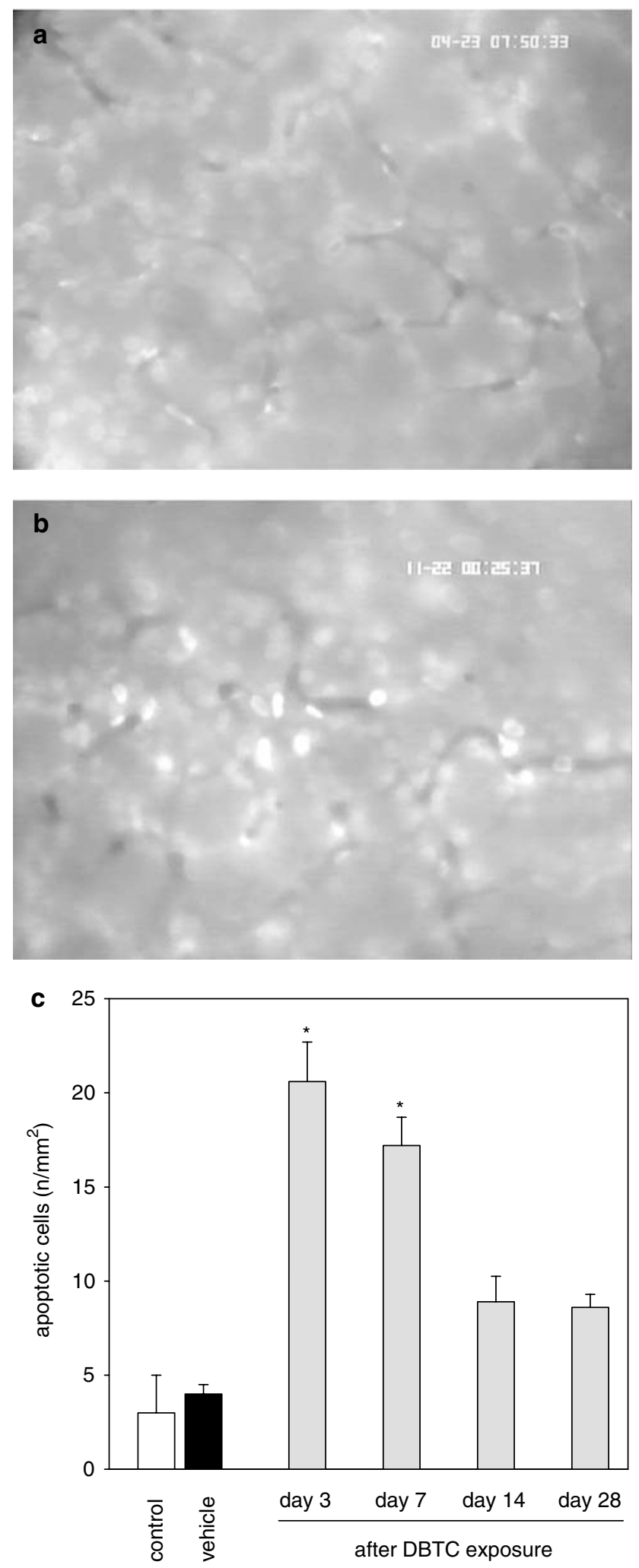

ical detection of active caspase-3 clearly revealed apoptosis of single acinar cells in DBTC pancreatitis. In parallel, necrotic cells were observed, underlining the fact that both modes of cell death may occur simultaneously in pancreatic tissue. Although pathways are not completely understood, there is emerging hypothesis that the early phase of both forms of cell death, that is, apoptosis and necrosis, involves mitochondrial dysfunction. ${ }^{29}$ While then high ATP levels might enable cells to undergo apoptosis, mitochondrial deenergization in the absence of caspase activation and ATP depletion shift cells from apoptosis to necrosis. ${ }^{30,31}$ Thus, the mixed and/or parallel form of acinar cell death, the so-called necrapoptosis ${ }^{25}$ might be best attributed to the noxious stimulus exerted by DBTC. ${ }^{16,17}$ This view is further underscored by the massive activation of leukocytes, interacting with the endothelial lining, infiltrating into tissue and leading to tissue damage, as indicated by the transient but marked rise of serum amylase and lipase activities.

The technique of intravital fluorescence microscopy employed in the present study proved itself useful in the simultaneous and serial in vivo analysis of the course of morphological, microvascular and cellular aspects and their relationship in consecutive stages of pancreatic inflammation and fibrosis. A remarkable inflammatory response and acinar cell death preceded loss of capillaries and pancreatic tissue fibrosis. This distinct time course underlines the importance of understanding the initial response to noxious stimuli, such as DBTC, in particular in terms of reversibility of morphological and functional destructuralization of the pancreas in future studies.

Due to enormous improvements in cell and molecular biological techniques, the pathobiology of collagen deposition and the definition of humoral factors and regulatory loops underlying both the amplification and degradation pathways of collagen during pancreatic fibrogenesis are reasonably well characterized. ${ }^{32-34}$ In this context, pancreatic stellate cells are considered to play a major role as primary cells regulating not only synthesis,,$^{1,3,4,35,36}$ but also

Figure 3 Representative intravital fluorescence microscopic images of bisbenzimide-stained pancreatic tissue after (a) vehicle-treatment or (b) at day 3 after DBTC exposure. Note the marked increase of acinar cells exhibiting apoptotic signs, namely condensation of nuclear chromatin, at day 3 after DBTC exposure. Original magnification $\times 807$. (c) Quantitative analysis of apoptotic cells within pancreatic tissue of animals at 3, 7, 14 and 28 days after intravenous DBTC exposure ( $n=7$ per time point). Animals that received equivalent volumes of solvent (vehicle, $n=12$ ) and animals without any intervention (control, $n=3$ ) served as controls. Using intravital fluorescence microscopy and a computer-assisted analysis system for off-line analysis, measurements were performed in ultraviolet epi-illumination after intravenous injection of bisbenzimide. For further details in analysis of apoptosis, see Materials and methods. Mean \pm s.e.m., ${ }^{*} P<0.05$ vs vehicle. 
However, injurious stimuli can activate pancreatic stellate cells, which then undergo functional and morphological changes with expression of cytoske-
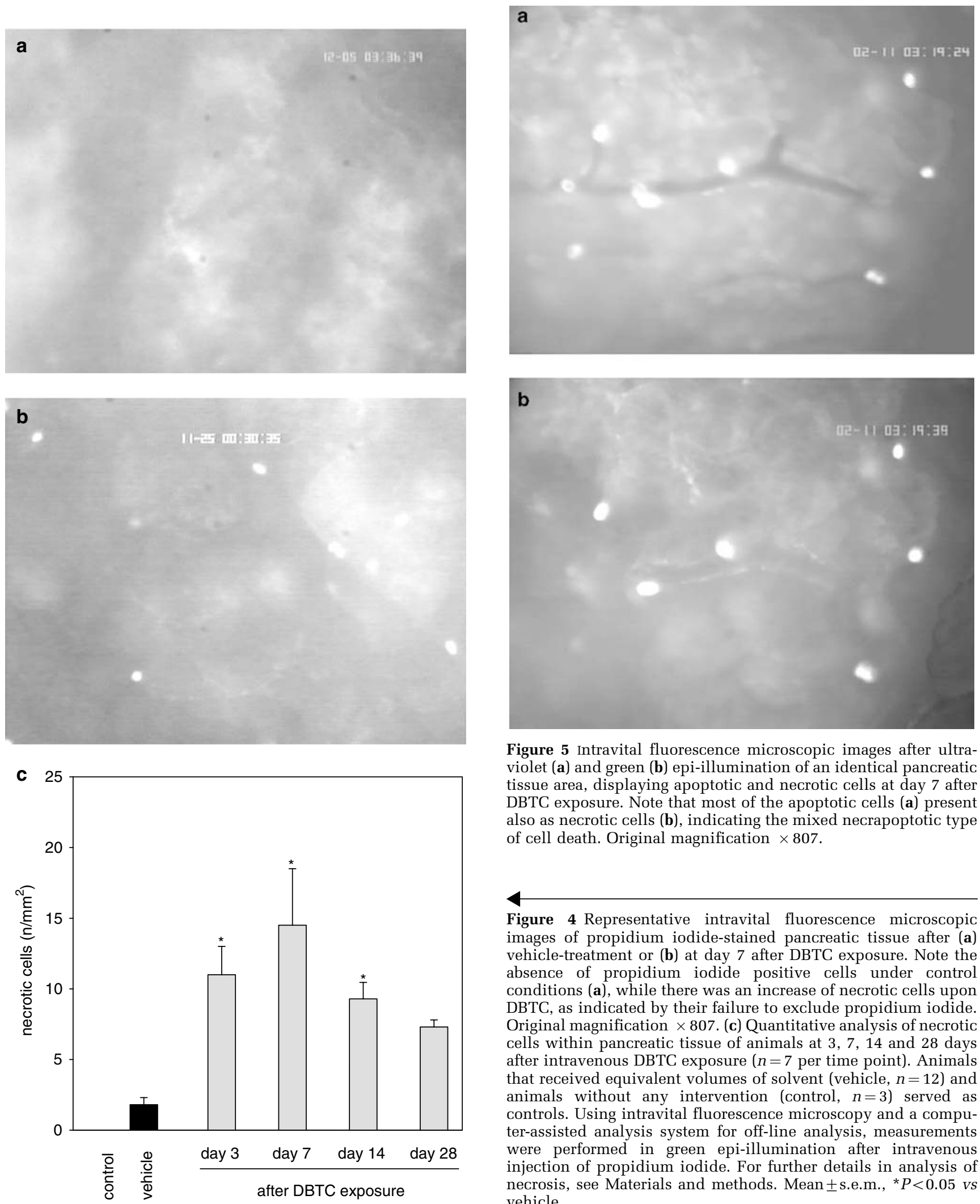

Figure 5 Intravital fluorescence microscopic images after ultraviolet (a) and green (b) epi-illumination of an identical pancreatic tissue area, displaying apoptotic and necrotic cells at day 7 after DBTC exposure. Note that most of the apoptotic cells (a) present also as necrotic cells (b), indicating the mixed necrapoptotic type of cell death. Original magnification $\times 807$.

Figure 4 Representative intravital fluorescence microscopic images of propidium iodide-stained pancreatic tissue after (a) vehicle-treatment or (b) at day 7 after DBTC exposure. Note the absence of propidium iodide positive cells under control conditions (a), while there was an increase of necrotic cells upon DBTC, as indicated by their failure to exclude propidium iodide. Original magnification $\times 807$. (c) Quantitative analysis of necrotic cells within pancreatic tissue of animals at 3, 7, 14 and 28 days after intravenous DBTC exposure ( $n=7$ per time point). Animals that received equivalent volumes of solvent (vehicle, $n=12$ ) and animals without any intervention (control, $n=3$ ) served as controls. Using intravital fluorescence microscopy and a computer-assisted analysis system for off-line analysis, measurements were performed in green epi-illumination after intravenous injection of propidium iodide. For further details in analysis of necrosis, see Materials and methods. Mean \pm s.e.m., ${ }^{*} P<0.05$ vs vehicle. 
leton protein SMA, similarily as this has been shown for hepatic stellate cells. ${ }^{38}$ Although not systematically analyzed in serial sections, Sirius red collagen staining was found in close vicinity with
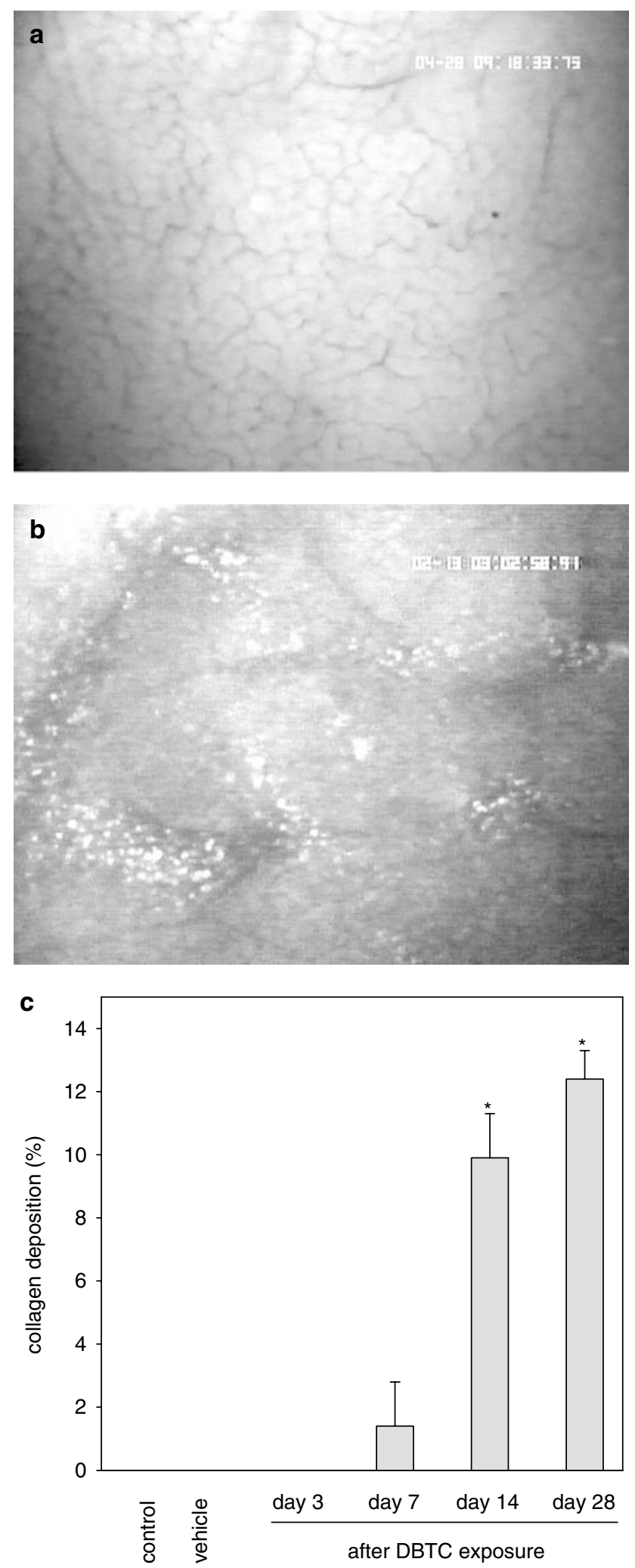
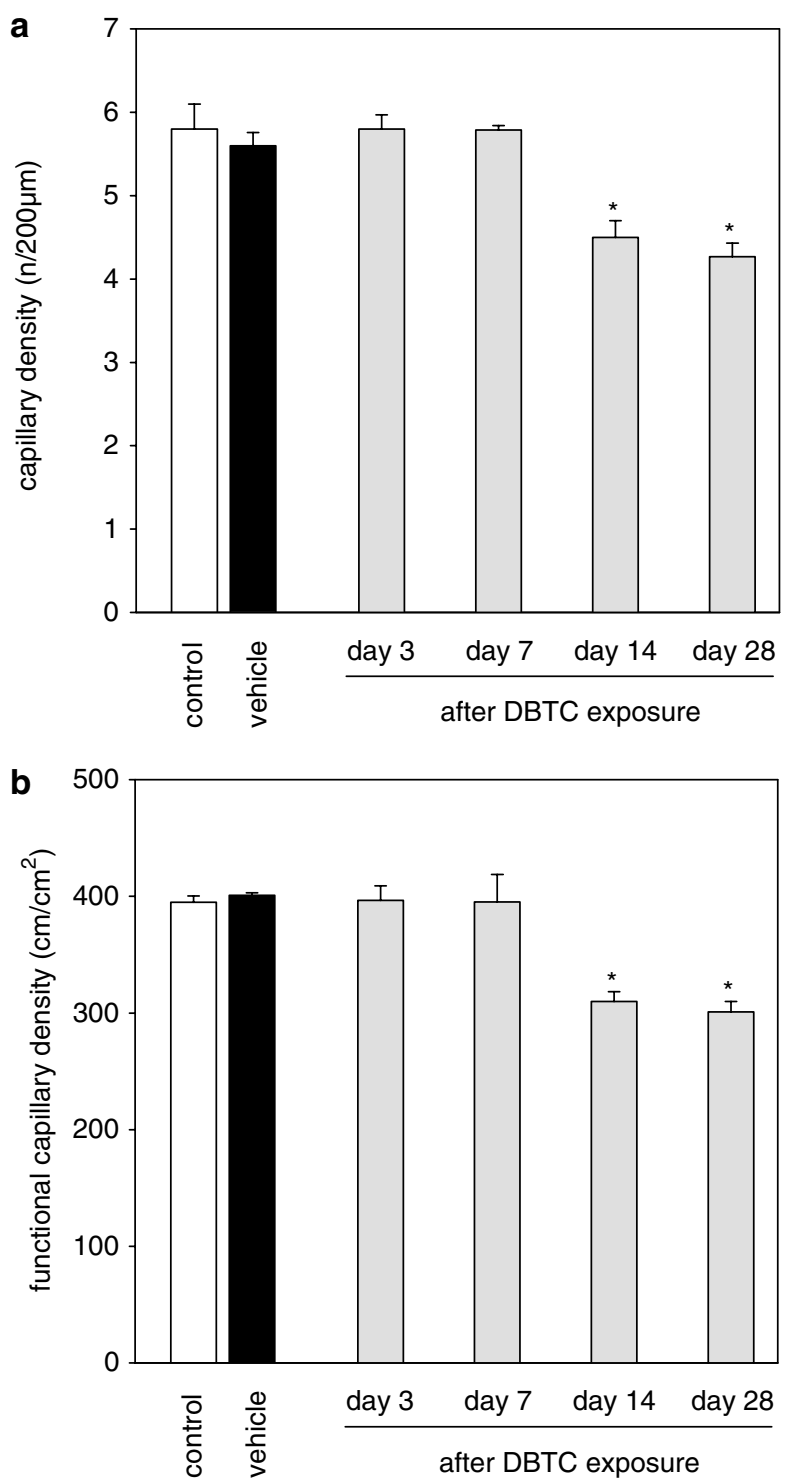

Figure 7 Quantitative analysis of capillary density (a) and functional capillary density (b) of exocrine pancreatic tissue in animals at 3, 7, 14 and 28 days after intravenous DBTC exposure ( $n=7$ per time point). Animals that received equivalent volumes of solvent (vehicle, $n=12$ ) and animals without any intervention (control, $n=3$ ) served as controls. Using intravital fluorescence microscopy and a computer-assisted analysis system for off-line analysis, measurements were performed in blue epi-illumination after injection of FITC-dextran for contrast enhancement of the microvasculature. Mean \pm s.e.m., ${ }^{*} P<0.05$ vs vehicle.

Figure 6 Representative intravital fluorescence microscopic images of autofluorescent collagen deposits in pancreatic tissue after (a) vehicle-treatment or (b) at day 28 after DBTC exposure. Original magnification $\times 208$. (c) Quantitative analysis of collagen deposition of pancreatic tissue in animals at 3, 7, 14 and 28 days after intravenous DBTC exposure ( $n=7$ per time point). Animals that received equivalent volumes of solvent (vehicle, $n=12$ ) and animals without any intervention (control, $n=3$ ) served as controls. Using intravital fluorescence microscopy and a computer-assisted analysis system for off-line analysis, measurements were performed in autofluorescent native pancreatic tissue upon blue epi-illumination. Mean \pm s.e.m., ${ }^{*} P<0.05$ vs vehicle. 

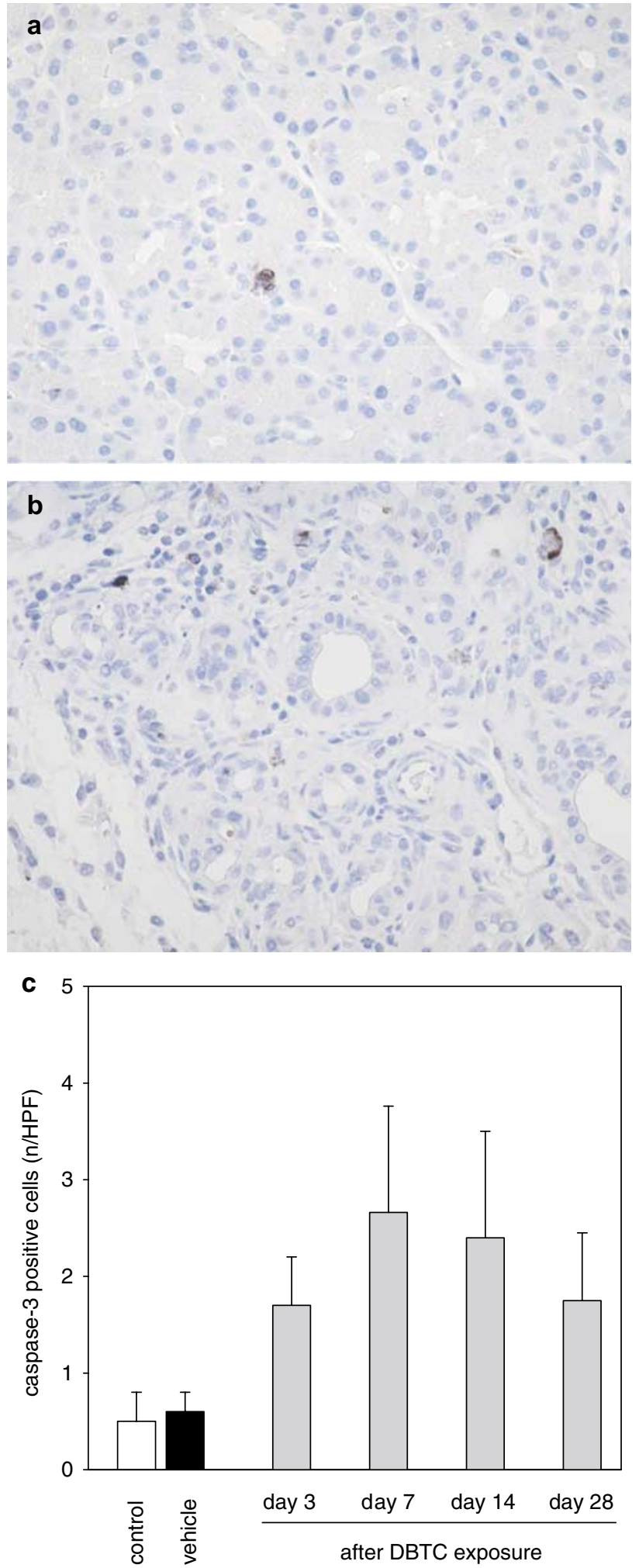

Figure 8 Representative images of caspase-3 immunohistochemical staining of pancreatic tissue after (a) vehicle-treatment or (b) at day 7 after DBTC exposure. Original magnification $\times 400$. (c) Quantitative analysis of caspase-3-positive cells (given as cells per HPF) in pancreatic tissue of animals at 3, 7, 14 and 28 days after intravenous DBTC exposure ( $n=7$ per time point). Animals that received equivalent volumes of solvent (vehicle, $n=12$ ) and animals without any intervention (control, $n=3$ ) served as controls. Mean \pm s.e.m.
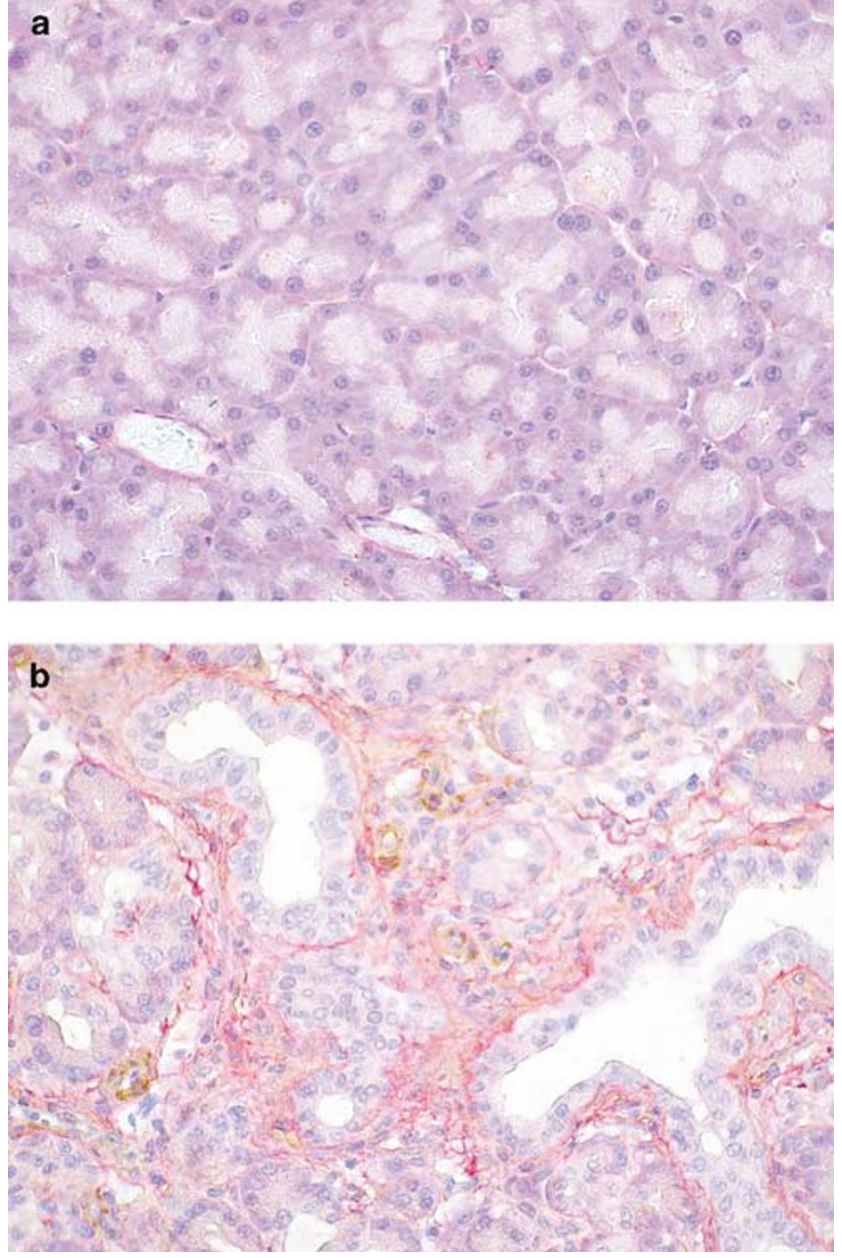

Figure 9 Representative images of dual staining for SMA plus Sirius Red in pancreatic tissue after (a) vehicle-treatment or (b) at day 14 after DBTC exposure. While there were no SMA-positive cells and only very faint Sirius Red collagen stain in pancreatic tissue of the vehicle-treated animal, Sirius red staining was associated with SMA-positive cells in the DBTC-exposed tissue, implying occurrence of pancreatic stellate cell activation during pancreatic fibrogenesis. Original magnification $\times 400$.

SMA positive cells in the present study. This finding corresponds with results from experimental rat pancreatic fibrosis, demonstrating that Sirius red staining was associated with SMA-positive cells, which in turn colocalized with procollagen alpha1 mRNA expression. ${ }^{4}$ Thus, present immunohistochemical analysis of pancreatic tissue may imply the activation of pancreatic stellate cells with subsequent extracellular matrix formation upon DBTC exposure. Heterogeneous distribution of autofluorescent collagen in vivo coincided with the histological picture of patchy fibrosis and SMA staining, implying a possible relationship between pancreatic stellate cell activation and collagen deposition. This speculation deserves confirmation by application of more appropriate procedures to exclusively study pancreatic stellate cells, which however exceed the scope of the present study. 
Collagen deposition, as determined by intravital microscopy, Ladewig as well as Sirius Red staining, and stellate cell activation, as determined by SMA expression, occur concomitantly with the reduction of capillary density. The present study demonstrates early onset with simultaneous and progredient development of microvascular, cellular and fibrotic alterations during the process of pancreatic fibrosis. The fact that, in dependence to the increase of autofluorescent collagen, capillary density is found progressively decreased indicates fibrosis-associated organ remodeling. Rarefication of microvessels comprises insufficient oxygen supply to tissue, as given by the decrease in functional capillary density, with the consequence of hypoxia-driven tissue collapse and organ atrophy.

The present study shows a comprehensive puzzle of the cellular, microvascular and morphological aspects of pancreatic fibrogenesis, characterized by an induction of single-cell apoptosis and necrosis, leukocyte activation and tissue infiltration finally with collagen deposition and tissue destruction. The herein used model reflects the pathogenesis of chronic pancreatitis and is thus ideally suited for evaluation of newly developed therapeutic strategies.

\section{Acknowledgements}

The study is supported by FORUN (\# 989022; Medical Faculty, University of Rostock). The authors kindly thank Dorothea Frenz, Department of Experimental Surgery, University of Rostock, for excellent technical assistance.

\section{References}

1 Bachem MG, Schneider E, Gross H, et al. Identification, culture, and characterization of pancreatic stellate cells in rats and humans. Gastroenterology 1998;115:421-432.

2 Wells RG, Crawford JM. Pancreatic stellate cells: the new stars of chronic pancreatitis? Gastroenterology 1998;115:491-493.

3 Apte MV, Haber PS, Darby SJ, et al. Pancreatic stellate cells are activated by proinflammatory cytokines: implications for pancreatic fibrogenesis. Gut 1999;44: 534-541.

4 Haber PS, Keogh GW, Apte MV, et al. Activation of pancreatic stellate cells in human and experimental pancreatic fibrosis. Am J Pathol 1999;155:1087-1095.

5 Foitzik T, Buhr HJ. New aspects in the pathophysiology of chronic pancreatitis. Chirurg 1997;68:855-864.

6 Kloppel G, Maillet B. Pathology of acute and chronic pancreatitis. Pancreas 1993;8:659-670.

7 Kloppel G, Maillet B. Chronic pancreatitis: evolution of the disease. Hepatogastroenterology 1991;38:408-412.

8 Kloppel G, Maillet B. The morphological basis for the evolution of acute pancreatitis into chronic pancreatitis. Virchows Arch A Pathol Anat Histopathol 1992;420:1-4.
9 Ammann RW, Heitz PU, Kloppel G. Course of alcoholic chronic pancreatitis: a prospective clinicomorphological long-term study. Gastroenterology 1996;111:224-231.

10 Vollmar B, Menger MD. Microcirculatory dysfunction in acute pancreatitis. A new concept of pathogenesis involving vasomotion-associated arteriolar constriction and dilation. Pancreatology 2003;3:181-190.

11 Klar E, Messmer K, Warshaw AL, et al. Pancreatic ischaemia in experimental acute pancreatitis: mechanism, significance and therapy. Br J Surg 1990;77: 1205-1210.

12 Menger MD, Bonkhoff H, Vollmar B. Ischemia-reperfusion-induced pancreatic microvascular injury. An intravital fluorescence microscopic study in rats. Dign Dis Sci 1996;41:823-830.

13 Schilling MK, Redaelli C, Reber PU, et al. Microcirculation in chronic alcoholic pancreatitis: a laser Doppler flow study. Pancreas 1999;19:21-25.

14 Kitagawa K, Takashima T, Matsui O, et al. Marked hypervascularity and early arteriovenous shunting with portal vein filling in chronic relapsing pancreatitis. Gastrointest Radiol 1986;11:56-60.

15 Pitkaranta P, Kivisaari L, Nordling S, et al. Vascular changes of pancreatic ducts and vessels in acute necrotizing, and in chronic pancreatitis in humans. Int J Pancreatol 1991;8:13-22.

16 Merkord J, Jonas L, Weber H, et al. Acute interstitial pancreatitis in rats induced by dibutyltin dichloride (DBTC): pathogenesis and natural course of lesions. Pancreas 1997;15:392-401.

17 Sparmann G, Merkord J, Jaschke A, et al. Pancreatic fibrosis in experimental pancreatitis induced by dibutyltin dichloride. Gastroenterology 1997;112: 1664-1672.

18 Swatland HJ. Fluorimetry of bovine myotendon junction by fibre-optics and microscopy of intact and sectioned tissues. Histochem J 1987;19:276-280.

19 Verbunt RJ, Fitzmaurice MA, Kramer JR, et al. Characterization of ultraviolet laser-induced autofluorescence of ceroid deposits and other structures in atherosclerotic plaques as a potential diagnostic for laser angiosurgery. Am Heart J 1992;123:208-216.

20 Vollmar B, Siegmund S, Menger MD. An intravital fluorescence microscopic study of hepatic microvascular and cellular derangements in developing cirrhosis in rats. Hepatology 1998;27:1544-1553.

21 Vollmar B, Preissler G, Menger MD. Hemorrhagic hypotension induces arteriolar vasomotion and intermittent capillary perfusion in rat pancreas. Am J Physiol 1994;267:H1936-H1940.

22 Vollmar B, Janata J, Yamauchi JI, et al. Attenuation of microvascular reperfusion injury in rat pancreas transplantation by L-arginine. Transplantation 1999; 67:950-955.

23 Schäfer T, Scheuer C, Roemer K, et al. Inhibition of p53 protects liver tissue against endotoxin-induced apoptotic and necrotic cell death. FASEB J 2003;17: 660-667.

24 Eipel C, Bordel R, Nickels RM, et al. Impact of leukocytes and platelets in mediating hepatocyte apoptosis in a rat model of systemic endotoxemia. Am J Physiol Gastrointest Liver Physiol 2004;286: G769-G776.

25 El-Gibaly AM, Scheuer C, Menger MD, et al. Improvement of rat liver graft quality by pi-fithrin- $\alpha$-mediated inhibition of hepatocyte necrapoptosis. Hepatology 2004;39:1553-1562. 
26 Takei Y, Marzi I, Gao WS, et al. Leukocyte adhesion and cell death following orthotopic liver transplantation in the rat. Transplantation 1991;51:959-965.

27 Klyscz T, Junger M, Jung F, et al. Cap image-a new kind of computer-assisted video image analysis system for dynamic capillary microscopy. Biomed Tech (Berl) 1997;42:168-175.

28 Klöppel G, Detlefsen S, Feyerabend B. Fibrosis of the pancreas: the initial tissue damage and the resulting pattern. Virchows Arch 2004, May 8 [Epub ahead of print].

29 Lee HC, Wei YH. Mitochondrial role in life and death of the cell. J Biomed Sci 2000;7:2-15.

30 Eguchi Y, Shimizu S, Tsujimoto Y. Intracellular ATP levels determine cell death fate by apoptosis or necrosis. Cancer Res 1997;57:1835-1840.

31 Nicotera P, Leist M, Single B, et al. Execution of apoptosis: converging or diverging pathways? Biol Chem 1999;380:1035-1040.

32 Kennedy RH, Bockman DE, Uscanga L, et al. Pancreatic extracellular matrix alterations in chronic pancreatitis. Pancreas 1987;2:61-72.
33 Jaster R, Sparmann G, Emmrich J, et al. Extracellular signal regulated kinases are key mediators of mitogenic signals in rat pancreatic stellate cells. Gut 2002;51: 579-584.

34 Phillips PA, McCarroll JA, Park S, et al. Rat pancreatic stellate cells secrete matrix metalloproteinases: implications for extracellular matrix turnover. Gut 2003;52:275-282.

35 Apte MV, Phillips PA, Fahmy RG, et al. Does alcohol directly stimulate pancreatic fibrogenesis? Studies with rat pancreatic stellate cells. Gastroenterology 2000;118:780-794.

36 Casini A, Galli A, Pignalosa P, et al. Collagen type I synthesized by pancreatic periacinar stellate cells (PSC) co-localizes with lipid peroxidation-derived aldehydes in chronic alcoholic pancreatitis. J Pathol 2000;192:81-89.

37 Apte MV, Haber PS, Applegate TL, et al. Periacinar stellate shaped cells in rat pancreas: identification, isolation, and culture. Gut 1998;43:128-133.

38 Friedman SL. Cytokines and fibrogenesis. Semin Liver Dis 1999;19:129-140. 